\title{
Consistency and Update in Mobile Overlay Networks
}

\author{
Zhou SU, Jiro KATTO, Yasuhiko YASUDA, and Yu CHEN, Members, IEEE \\ ContactEmail:zhousu@asagi.waseda.jp
}

\begin{abstract}
With the development whereby mobile content distribution systems can manage dynamically changing files, an important issue to be resolved is consistency management, which means the cached replicas on different mobile sites must be updated if the originals change. This paper is to design an integrated consistency-control algorithm for mobile contents delivery network (M-CDN) to enhance the efficient utilization of network resource and support the client mobility. Firstly, by carrying out an analysis of mobile surrogates' characteristics, for a given content which has been changed on its original node, only a limited number of its replicas instead of all replicas are updated. Secondly, if a replica has been selected for update, the latest version will be sent from an algorithm-decided site instead of from its original server. Simulation results show that the proposal outperforms other conventional methods.
\end{abstract}

Index Terms - Mobile contents delivery, Consistency Algorithm, Web Performance, Network Traffic

\section{INTRODUCTION}

W the rapid developments of wireless communication technologies, a mobile contents delivery network has appeared to facilitate a set of moving nodes to communicate with each other via short-range wireless transmission protocols such as IEEE 802.11, Blue-tooth or Ultra Wide Band (UWB). In the mobile CDN, a moving node receives information from its neighbors, or from remote nodes by multi-hop transmission relayed by intermediated moving nodes [1][2].

This technology has attracted many attentions and applications: for example, the Nokia mobile server has been proposed to make a web-server running on a mobile phone accessible from the Internet using any web browsers. Furthermore, the Helix mobile server led by Real Networks is optimized for delivery of all major file formats to any wireless network, with the broadest support for mobile delivery standards. However, although the above companies advocate

Zhou SU, Jiro KATTO, Yasuhiko YASUDA are with Department of Computer Science, Waseda Umiversity, Okubo 3-4-1, Shinjuku-ku, Tokyo 169-8555, JAPAN

Yu CHEN., is with Department of Electrical and Computer Engineering State University of New York - Binghamton Binghamton, NY 13902, USA their wireless support for contents delivery, their technical details on how to keep the content consistency have not yet clarified nor verified.

In a mobile $\mathrm{CDN}$, an important issue to be resolved is consistency management, which means the cached replicas on different sites must be updated if the originals change. If a requested object can be provided to the user with the latest version, the user will not need to contact the remote origin server to get the updated object. Therefore, the appropriate consistency control of server replicas benefits content providers by reducing latency for their clients, and benefits ISPs by reducing bandwidth consumption and transmission cost.

The conventional consistency methods for wired networks could not be efficiently carried out in mobile environment. It is because: in contrast to conventional CDN systems in wired networks that consist of surrogates, mobile CDN are subjected to the limitations of long-lived nature of typical multimedia sessions, wireless bandwidth, and the dynamically changed network topology. Challenges arise in how to improve the data consistency in a mobile CDN.

In this paper, we therefore propose an optimal algorithm for controlling Web consistency in mobile contents delivery overlay.

Firstly, we make an analysis on the Web access distribution in mobile contents delivery network. When a given content changes at its original server, a consistency priority, which is proposed based on the analysis of mobility, will be calculated. For a given content's each replica over the whole overlay network, only when its priority is beyond the threshold, the replica of content at this surrogate will be updated.

Secondly, the process of the delivery of contents in M-CDN is discussed. After considering the available sessions, received signal strength and user satisfaction degree, an update priority is proposed, where the surrogate (instead of the original server) with the highest update priority will provide the requested contents to the client.

This paper is organized as follows: in Sect.II, related work with regard to mobile contents delivery and consistency management algorithms is reviewed. Sect.III presents mathematical analyses of contents selection and surrogate update. And our proposed algorithm is also presented in Sect.IV. In Sect.V, simulation results are given and conclusions 
are presented in Sect.VI.

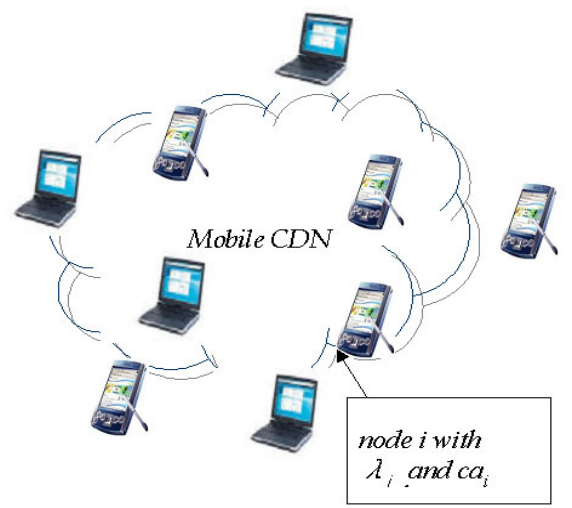

Fig1: Mobile Dynamic Contents Delivery Overlays

\section{PREVIOUS WORK}

Content distribution networks (CDNs) have been playing an important role in managing content distribution to large numbers of users in wired networks. However, unlimited in the wired network, with the development of wireless technology, the demands for mobile content delivery to dynamically changing client, has appeared in recent years.

[11] talked about the importance of the Web consistency and also proposed a method of Consistency-preserving Caching for Dynamic Database Content. However, the above work doesn't support the wireless communication. In [12], an optimized query planning of continuous aggregation queries in dynamic data dissemination networks was presented. This method of query execution can be implemented using schemes similar to that used in CDNs. However, how to optimally control the query to improve consistency is still not resolved.

[4] proposed a mobile surrogate-selection scheme that can significantly reduce the number of server-handoffs necessary for mobile multimedia contents delivery via a high density M-CDN. In this method, all surrogates are divided into 2 tiers: Lower-tier servers are topologically closer to the clients, and hence can deliver better QoS in terms of end-to-end delay and jitter. On the other hand, higher tier servers have a larger coverage area and hence their clients incur fewer server handoffs. However, the criterion for dividing surrogates into different tiers is still not clearly presented. Furthermore, the cooperation among different tiers is not talked about.

[1] proposed a M-CDN model for the replication in enterprise networks, which takes client demand variations into account to dynamically add/remove content replicas to/from the network in order to minimize the total traffic over the backbone. It provided two solutions: an offline optimal and a heuristic online solution. Although the proposed model is helpful to contents replication, its analysis is only based on the network traffic. The corresponding analysis about network bandwidth and topology should be carried out.

In [8], it concluded that using the fixed caching proxies to serve mobile nodes may defeat the entire purpose of using caches for traffic localization, therefore it is important that the mobile node can switch to appropriate caching proxy as it moves. Their results showed that delay experienced by mobile nodes could be significantly reduced if mobile surrogate keeps switching to nearest caching proxy. Thought this work proved that keeping the replica on different proxies is helpful, how to keep the consistency of the replicas on different proxy has not been mentioned.

A cooperative caching and data consistency scheme [2], called Proximity Regions for Caching in Cooperative (PReCinCt), has been proposed to support scalable data retrieval in large-scale Mobile Peer to Peer (MP2P) networks. This cooperative caching scheme enables peers in a region to share their data, thus providing a unified view of the cache. This helps in alleviating the message latency and limited accessibility problems in MP2P networks. The caching scheme considers data popularity, data size and region-distance during replacement to optimize cache content of peers. The above method used a parameter called Time-to-Refresh (TTR) to keep the consistency among the replicas, however, how to decide an optimal value of Time-to-Refresh (TTR) was not resolved.

In Propagation method, the updated version of a document is delivered to all copies whenever a change is made to the document on the origin server. Although the copies always keep the latest version of the originals by the Propagation, this method may generate significant levels of unnecessary traffic if documents are updated more frequently than accessed.

In Invalidation [7], an invalidation message is sent to all copies when a document is changed on the origin server. This method does not make full use of the delivery network for content delivery and each replica needs to fetch an updated version individually at a later time. Therefore, the user-delay may get worse if a frequently accessed document can not be updated on time.

We ourselves proposed an integrated pre-fetching and searching algorithm for mobile P2P model, where the query can be efficiently used [9]. We also presented a scheme to manage web consistency in a contents delivery network, in which all nodes are wired without mobile contents [10]. The former is limited to P2P network and the later is designed for wired network, which are both different from the proposal in this paper.

\section{THEORY ANALYSIS}

\section{A. Contents Selection}

We consider an overlay network that has $K$ mobile nodes. Each node $i(i=1, \ldots, K)$, has a $\lambda_{i}$ (bytes/second) which denotes an aggregate request rate from clients to the server.

As for the contents, we assume that there are $J$ different contents in our mobile CDN. A parameter $P_{j}$ defines the request probability for content $j$, with a data size $B_{j}$.

According to the Zipf distribution which the distribution of many Web accesses follows, the probability that the content $j$ is requested can be obtained as follows:

$$
P_{j}=\frac{V}{w_{j}^{\alpha}}
$$


where $V, \alpha$ are parameters of the Zipf distribution, and $w_{j}$ is the ranking of request times.

We assume that there is a Request Routing (RR) function in each node. The RR maintains the residence time value $r_{q, i}$ for each client $q$ in node $i$ 's zone.

Then, the relative residence of a client with a node $i$ can be

$$
r_{i}=\frac{\sum_{q} r_{q, i}}{\sum_{i} \sum_{q} r_{q, i}}
$$

Assume that a client firstly enters in the area of node $i$ and then requests contents $j$, we can get the probability that the content $j$ is requested from node $i$ to be:

$$
\theta_{i, j}=\frac{V}{w_{j}^{\alpha}} \cdot \frac{\sum_{q} r_{q, i}}{\sum_{i} \sum_{q} r_{q, i}}
$$

We call the above one consistency priority $\theta_{i, j}$. Note that this priority can support node-mobility since both the residence time and the entering area are taken into consideration. The usage of it will be introduced in later sections.

\section{B. Surrogate Update}

In a conventional method, if a surrogate decides to fetch the latest version of a modified document, it will contact the modified document's original server to fetch its latest version. However, this method causes a bad load balance if all requests going to the original server. Furthermore, fetching the contents from the original server ( which is far from the client ) also increased the extra network traffic.

To select a proper surrogate to provide the latest version of the requested content to the client becomes an important issue. Here, we think the proper surrogate should at least satisfy the following three requirements: 1) be available: The surrogate should have enough free sessions to receive the request when a latest version is wanted from the client. 2) be reachable: The received signal strength (RSS) of the surrogate should be strong enough to let the requested content arrive at the client. 3) be satisfied: The client should be satisfied with the quality (such as user delay) after receiving the updated content from the surrogate.

We make analyses of the above requirements as follows:

\section{1) Analysis of the Availability}

Assume that the node $i$ has a coverage area denoted by the radius of $c a_{i}$ and its distance between node $k$ is $D_{i, k}$.

For each node $k$, if it is in the coverage area of node $i$, then we can get:

$$
\begin{aligned}
& D_{i, k} \leq c a_{i} \\
& k=\{1,2, \ldots K\} \& k \neq i
\end{aligned}
$$

Let $Z_{k}$ denote the capacity (the number of simultaneous sessions) of node $k$, and $Z$ is the number of total sessions happened in the whole network within a testing past period.
Then the average sessions happened in node $k$ during the past period can be:

$$
\left.\left(\lambda_{k} / \sum_{k} \lambda_{k}\right) \cdot Z\right)
$$

A free-degree is defined as follows to show the number of free sessions in node $k$.

$$
f_{k}=\left(Z_{k}-\left(\lambda_{k} / \sum_{k} \lambda_{k}\right) \cdot Z\right) / Z_{k}
$$

\section{2) Analysis of the Reachable Ability}

Assume that the received signal strength (RSS) of the node $k$ is $R S S_{k}$. The probability that its signal is better than the threshold is:

$$
\begin{aligned}
& l_{k}=P\left\{R S S_{k} \geq \text { Thresh }\right\} \\
& =P\left\{R S S_{k} \mid R S S_{k} \geq \text { Thresh }\right\} / T
\end{aligned}
$$

where Thresh is the set threshold and $T$ is the total detecting times within the testing period.

\section{3) Analysis of the Satisfaction}

Recent study [6] shows that user $i$ 's satisfaction (or utility) with receiving the requested content $j$ depends on the user-delay $\left(t_{k(i . j)}\right)$ to obtain content $j$ from surrogate $k$.

$$
S_{k(i, j)}=e^{-r \cdot t_{k(i, j)}}
$$

Here, $r$ is a satisfaction parameter that is varied according the user's sensitivity.

As the user-delay is very important to reflect the user's satisfaction, we calculate the user-delay, which is caused during the delivery from a surrogate to the client (requested surrogate) as follows.

Assume that content $j$ is originally stored in server $o(j)$ and $C_{k(i, j), i}$ is the average bandwidth (per hop) during the path from surrogate $k(i, j)$ to surrogate $i$. Here, surrogate $i$ is the node where the client sent the request, while surrogate $k(i, j)$ is a node which has the latest version of content $j$.

Then, when a request for content $j$ happens at surrogate $i$ and surrogate $k(i, j)$ sends the latest version to satisfy this request, the user delay during the delivery from surrogate $k(i, j)$ to surrogate $i$ is given by

$$
t_{k(i, j), i, j}=\frac{1}{\Lambda} \lambda_{i} \cdot B_{j} \cdot P_{j} \cdot D_{k(i, j), i} / C_{k(i, j), i}
$$

Here, $C_{k(i, j), i}$ means the shortest distance (hop count) surrogate $k(i, j)$ to surrogate $i$.

If we continue to define:

$$
G_{j}=\frac{1}{\Lambda} \cdot B_{j} \cdot P_{j}
$$




$$
U_{k(i, j), i}=D_{k(i, j), i} / C_{k(i, j), i}
$$

Eq.(9) becomes as follows:

$$
t_{k(i, j), i, j}=\lambda_{i} \cdot G_{j} \cdot U_{k(i, j), i}
$$

Then, we can obtain the user's satisfaction degree $\left(S_{k(i, j)}\right)$ when surrogate $k(i, j)$ provides the latest version of content $j$ to surrogate $i$ (where the user is )

$$
\begin{aligned}
& S_{k(i, j)}=e^{-r \cdot t_{k(i, j)}} \\
& =e^{-r\left(\lambda_{i} \cdot G_{j} \cdot U_{k(i, j), i}\right)}
\end{aligned}
$$

For simplicity, we use $S_{k}$ to represent $S_{k(i, j)}$ in the following section.

\section{4) Node Update Priority}

We define an index for update as follows:

$$
\Delta_{k}=f_{k} \cdot l_{k} \cdot S_{k}
$$

Let $X_{i, j}$, be a parameter which takes a binary value of $X_{i, j}=1$ (if content $j$ is stored in node $i$ )

$X_{i, j}=0$ (otherwise)

Then, we can get a matrix $\boldsymbol{X}$ of which element is $X_{i, j}$, which represents a placement pattern of contents.

For the mobile surrogates, which are in the coverage of node $i$, and also have the replica of content $j$, we can select the mobile surrogate $k$ with the maximum update index $\left(\Delta_{k}\right)$ by

$$
\left\{\begin{array}{c}
i \neq k \quad \& X_{k, j}=1 \\
D_{i, k} \leq c a_{i} \\
\max \left(\Delta_{k}\right)
\end{array}\right.
$$

\section{PROPOSAL}

We present our algorithm as follows:

- Step1: Update Selection

When a given content $j$ changes at its original server $o(j)$, a consistency priority $\theta_{i, j}$ will be calculated according to Equa.3. For content $j$ 's each replica $\left(X_{i, j}=1\right)$ over the whole mobile network, only when its priority $\theta_{i, j}$ is beyond the threshold, the replica of content $j$ at mobile surrogate $i$ will be updated.

Otherwise, this replica will not be updated until a new request for content $j$ happens at mobile surrogate $i$ next time.

Therefore, when a given content $j$ is changed at its original server, not all its replicas $\left(X_{i, j}=1\right)$ over all overlay network will be updated according to the analysis of Web access distribution.

- Step2: Highest Satisfation Update
Assume that there are $K\left(X_{k, j}=1, k=\{1, \ldots, K\}\right)$ replicas of content $j$ slected to be updated, for a replica at mobile surrogate $k$, an update priority $\Delta_{k}$ will be calculated according to Equa.16.

The latest version of content $j$ will be sent to the surrogate (which replica need to be replicated) from mobile surrogate $k$ with the maximum $\Delta_{k}$.

Therefore, the latest version will be sent from an algorithm-decided site instead of its original server resulting in the highest satisfaction.

\section{Simulation Results}

In the simulation scenario the mobile CDN comprises 21 nodes arranged by the Power-Law distribution [3]. For each node, the radius of coverage area is $2 / 3$ of its longest distance to other nodes. $R S S$ is set to be one of values of the set $\{1,2,3\}$, where 3 means the strongest value of RSS. The capacity of each node is varied between 50 to 300 simultaneous sessions [4].

As for the request, it arrives according to a Poisson process. All clients' requests are always redirected to the closest server. Residence time is decided at random from 1 minute to 2.5 minutes at random [4].

There are 1000 different contents in the network. The Web request for each content is decided by this Zipf distribution with a Zipf parameter 0.6[5]. The update rate of a given object is decided at random. The total request times in the simulations are 100000 .

There are 3 replication algorithms we will study:

\section{Invalidation Policy \\ TTR Policy \\ Proposal}

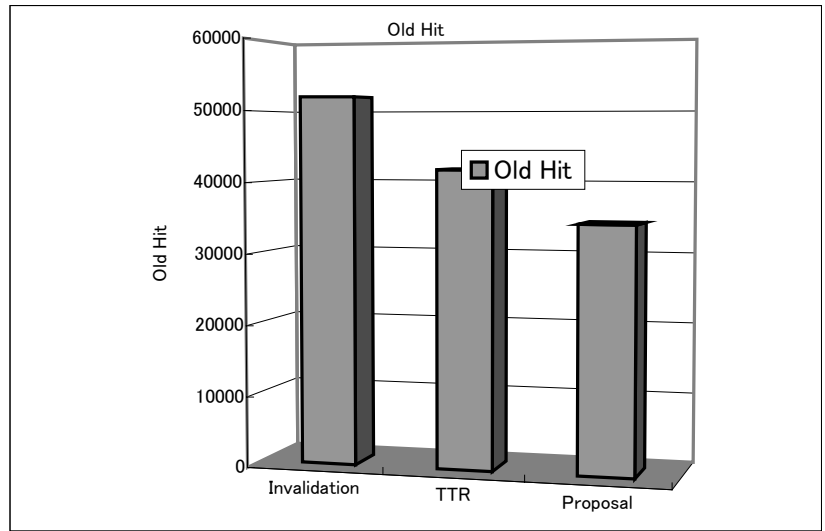

Fig.2: Comparison of Old Hit between Invalidation and Proposal.

It can be found that our proposal can get better Old Hit than the Invalidation in Fig.2. The reason is that: the proposal selects parts of (not all of) contents to be updated as whenever its original is changed on the original site. Then the Old Hit can be improved. On the other hand, why the proposal outperforms $T T R$ is because the proposal uses Residence time to decide the consistency priority. However, TTR only uses update-interval 
which does not support the mobility of nodes in M-CDN.

In Fig.3, the proposal can also reduce network traffic most than other two algorithms. In the proposal, the latest version the requested data is sent from the surrogate decided by the algorithm. This selected surrogate, which is in the coverage area of the mobile node (client), can provide high user's satisfaction with low user-delay. Since it is not necessary to fetch the date from the original server (which is necessary both in Invalidation and TTR), which is far way from the node where the request happens. Compared with the Invalidation, the TTR causes worst traffic since it fetch the data itself from the original server while the Invalidation only send an Invalidation information.

Finally, we conclude that our proposal can get better Old Hit and less Network traffic than the Invalidation and the TTR.

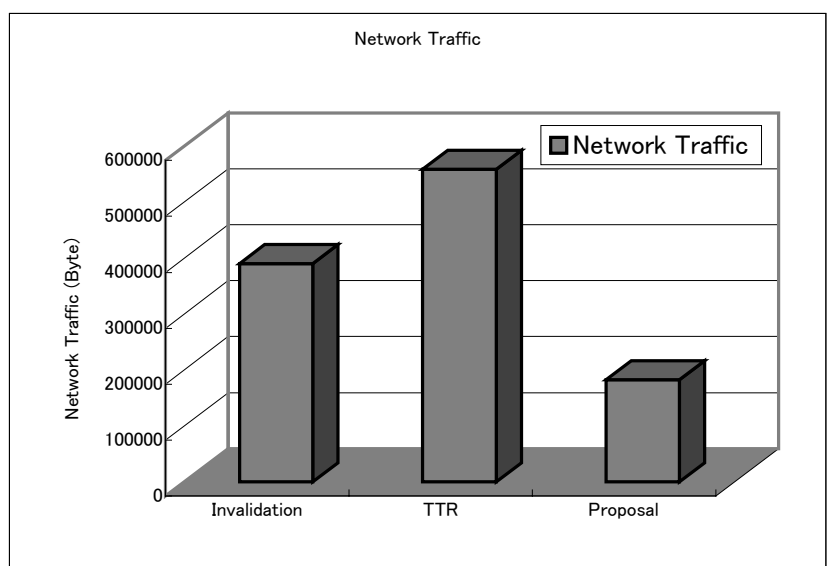

Fig.3: Comparison of Network Traffic between Invalidation and Proposal.

\section{CONCLUSIONS AND FURTHER WORK}

In this paper, based on mathematical analysis we proposed an integrated algorithm to manage the consistency of different replicas in M-CDN. On one hand, the proposal can decide whether a replica should be updated when its original content changes. On the other hand, if a replica has been decided for update, from which surrogate the latest version should be sent to the client can also be determined. We compared our proposal with other conventional methods by simulations.

More simulation results will be carried out from the practical viewpoint. Furthermore, theoretical modeling for consistency control should be expanded and sophisticated.

\section{REFERENCES}

[1] Wagner M. Aioffi, Geraldo R. Mateus, Jussara M. de Almeida, and Antonio A. F. Loureiro. "Dynamic Content Distribution for Mobile Enterprise Networks". IEEE Trans on Selected Areas in Communications, NO. 10, Oct 2005

[2] H.Shen, M.S.Joseph, M.Kumar, and S.K. Das, "PReCinCt: A Scheme for Cooperative Caching in Mobile Peer-to-Peer Systems", Proceedings of the 19th IEEE International Parallel and Distributed Processing Symposium, 2005

[3] G. Antoniol, G. Casazza, G. Di Lucca, M. Di Penta, and E. Merlo, "Predicting Web Site Access: An Application of Time Series", Proceedings of IEEE the third International Workshop on Web Site Evolution, Florence, Nov.2001.

[4] M. Tariq, R. Jain and T. Kawahara: "Mobility Aware Server Selection for Mobile Streaming Multimedia Content Distribution Networks, " Proceedings of International Web Caching Workshop (IWCW), Sep. 2003.

[5] L. Breslao, P. Cao, L. Fan, G. Phillips, and S. Shenker "Web Caching and Zip-like Distributions: Evidence and Implications" Proceedings of IEEE INFOCOM'99, New York, Apr. 1999.

[6] K.Nomura, K.Yamori, E.Takahashi, T.Miyoshi, and Y.Tanaka, "Waiting Time versus Utility to Download Images," Proceedings of 4th Asia-Pacific Symposium on Information and Telecommunication Technologies (APSITT 2001), Kathmandu, Nepal / Atami, Japan

[7] P. Cao and C. Liu, "Maintaining Strong Cache Consistency in theWorld-Wide Web," Proceedings of the 17th Int'l Conf. Distributed ComputingSystems, May.1997.

[8] M. Tariq and A. Takeshita, "Management of cacheable streaming multimedia content in networks with mobile hosts," Proceedings of IEEE GLOBECOM, Taipei, Taiwan, 2002, pp. 2257-2258.

[9] Zhou SU, Jiro KATTO and Yasuhiko YASUDA, "An integrated Retrieval and Pre-fetching algorithms for Segmented Streaming in Mobile Peer-to-Peer Networks" Proceedings of IEEE Cluster2005, September, Boston, Massachusetts, USA, 2005

[10] Zhou SU, Jiro KATTO and Yasuhiko YASUDA, "Scalable Maintenance for Strong Web Consistency in Dynamic Content Delivery Overlays", Proceedings of 2007 IEEE International Conference on Communications (ICC 2007), Scotland, Jun, 2007

[11] Niraj Tolia and M. Satyanarayanan, "Consistency-preserving Caching of Dynamic Database Content", Proceedings of WWW2007, Banff, Canada, May, 2007

[12] Rajeev Gupta, Krithi Ramamritham "Optimized Query Planning of Continuous Aggregation Queries in Dynamic Data Dissemination Networks", Proceedings of WWW2007, Banff, Canada, May, 2007 